\title{
Shortened left ventricular filling time in dilated cardiomyopathy: additional effects on heart rate variability?
}

Mouanodji Mbaissouroum, Christine O’Sullivan, Stephen J Brecker, Han B Xiao, Derek G Gibson

\begin{abstract}
Objective-To assess possible mechanical influences underlying the reduced heart rate variability in patients with dilated cardiomyopathy.

Design-Comparison of standard nonspectral indices of heart rate variability with echocardiographic Doppler measures of left ventricular function in patients and normal controls.
\end{abstract}

Patients-20 patients with dilated cardiomyopathy and 15 normal subjects of similar ages were studied.

Methods-Standard non-spectral indices of heart rate variability were measured over 24 hours. These were correlated with left ventricular cavity size, shortening fraction, and isovolumic relaxation time measured by $M$ mode echocardiography, and the duration of functional mitral regurgitation and left ventricular filling time assessed by continuous wave Doppler.

Results-Mean RR interval and estimates of short term variability (root mean square difference of successive $R R$ intervals, proportion of adjacent $R R$ intervals $>50 \mathrm{~ms}$ different, and SD indices) were not different from normal. The overall mean (SD) of the RR interval (65 (35)), and SD of five minute mean RR intervals (55 (30)), however, were reduced compared with normal values (115 (40) and 105 (45); $p<0.01$ for both). Neither correlated with left ventricular cavity size or shortening fraction, but both were strongly related to left ventricular filling time (coefficient of variation, $r=0.82$ and $r=0.81$ respectively). Correlation persisted when the SD was corrected for $R R$ interval $(r=0.69)$ although this correlation was not found in the controls. In individual patients, the difference between RR interval at the time of echo and minimum value during the 24 hours, a measure of ability to increase heart rate, also correlated closely with filling time $(r=0.92)$.

Conclusion-As the duration of functional mitral regurgitation is effectively fixed, its presence can limit the time available for left ventricular filling in dilated cardiomyopathy when heart rate is high. This may become the mechanism by which maximum heart rate is set, becoming fixed to optimise cardiac output. This manifestation of dilated cardiomyopathy may be an important factor in reduction of heart rate variability in these patients.

(Br Heart $\mathcal{f} 1993 ; 69: 327-331)$

Heart rate variability may be strikingly reduced in patients with left ventricular disease in the setting both of acute myocardial infarction, ${ }^{12}$ and chronic heart failure. ${ }^{3-5}$ This reduced variability has been attributed to autonomic dysfunction, ${ }^{367}$ and its presence has proved a sensitive predictor of sudden death. ${ }^{28}$ We have previously noted that in some patients with dilated cardiomyopathy, left ventricular filling time may be very short and depend critically on heart rate. ${ }^{9}$ We were thus interested to see whether this additional constraint might alter heart rate variability, based on the possibility that in some patients reduced heart rate variability might have a mechanical component rather than a purely neurogenic basis.

\section{Patients and methods}

We studied 17 men and three women with a mean (SD) age of 57 (14) years with dilated cardiomyopathy, defined as a left ventricular end diastolic dimension measured by $\mathbf{M}$ mode echocardiography, of $6.5 \mathrm{~cm}$ or more, and shortening fraction of $20 \%$ or less. All the patients were in sinus rhythm during the study. None was taking a $\beta$ adrenergic blocking drug and none had diabetes that required treatment with insulin or oral hypoglycaemic agents. We also studied a control group of 15 subjects, mean (SD) age $53(10)$ years, who had been referred with a non-specific history of palpitations, and in whom no significant disturbance of rhythm had been found on Holter monitoring. Clinical examination, chest $x$ ray, film, electrocardiogram, and standard echocardiography were all within normal limits in these subjects.

\section{ECHOCARDIOGRAMS}

$M$ mode echocardiograms, guided by the cross sectional display, were recorded photographically with a simultaneous electrocardiogram (lead 2) and phonocardiogram at 10 $\mathrm{cm} . \mathrm{s}^{-1}$ The internal dimension of the left ventricle was measured at end diastole (the onset of the QRS complex) and end ejection (synchronous with the aortic component of the second heart sound $\left(A_{2}\right)$ from leading edges. The identity of $\mathrm{A}_{2}$ was routinely checked against the aortic echogram. Isovolumic 
relaxation time was measured as the time interval from $A_{2}$ to mitral cusp separation as measured from the mitral echogram.

Continuous wave Doppler signals were recorded from the apex, with a $2 \mathrm{MHz}$ pencil transducer and a Doptek spectral analyser, again with simultaneous electrocardiograph and phonocardiogram at a paper speed of 10 $\mathrm{cm} . \mathrm{s}^{-1}$. Signals were taken from the mitral valve to record functional regurgitation. From this we found the duration of the valvar regurgitation signal itself, and also the filling period, which is the difference between the $R R$ interval and the duration of the regurgitant signal. ${ }^{9}$

\section{AMBULATORY ELECTROCARDIOGRAMS}

Three channels of electrocardiogram were recorded over 24 hours on a Marquette Series 8500 recorder with a standard 60 minute cassette tape. The patients kept a diary of the day's events, and were encouraged to continue with their normal activities. Tapes were played back through a Marquette Laser SXP analyser for analysis. All tapes were checked manually to clarify the origins of unlabelled beats. All coupling intervals containing noise or ectopic beats, atrial or ventricular, were excluded.

\section{ANALYSIS}

We used standard non-spectral indices of heart rate variability, ${ }^{135}$ defined by the Marquette programme:

(a) the $\mathrm{SD}$ about the mean $\mathrm{RR}$ interval throughout the 24 hours.

(b) The SD of the five minute means (SDANN index).

This procedure effectively inserts a low frequency filter and is thus a measure of how much heart rate varies over each five minute period throughout the 24 hours.

(c) The mean of the five minute SDs (SD index).

(d) The proportion of beat to beat differences in $R R$ interval $>50 \mathrm{~ms}$ computed from triplets of normally conducted beats (PNN 50 index).

(e) The root mean square difference between successive $R R$ intervals (RMSSD index), again computed from triplets of normally conducted beats. The difference between the two RR intervals is squared and summed, and the mean value taken. The three last indices are measurements of the higher frequencies of heart rate variability.

Table Heart rate variability

\begin{tabular}{llll}
\hline & $\begin{array}{l}\text { Normals } \\
(n=15)\end{array}$ & $\begin{array}{l}\text { Patients } \\
(n=20)\end{array}$ & $p$ Value \\
\hline RR interval (ms) & $790(100)$ & $730(200)$ & NS \\
SD of RR interval (ms) & $115(40)$ & $65(35)$ & $<0.01$ \\
SDANN index (ms) & $105(45)$ & $55(30)$ & $<0 \cdot 01$ \\
RMSSD index (ms) & $25(8)$ & $20(14)$ & NS \\
PNN 50 index (\%) & $6.3(5.6)$ & $3.7(5 \cdot 8)$ & NS \\
SD index & $46(13)$ & $27(19)$ & $<0.05$ \\
Coefficient of variation of & $0 \cdot 15(0.046)$ & $0.084(0.030)$ & $<0.01$ \\
RR interval & & & \\
\hline
\end{tabular}

Values are means (SD); PNN 50 index, proportion of adjacent RR intervals more than $50 \mathrm{~ms}$ different; RMSSD index, root mean square difference of successive RR intervals; SDANN different; RMSSD index, root mean square
index, SD of 5 minute mean $R R$ intervals. (f) To allow for the effect of RR interval as a possible confounding variable, we calculated a coefficient of variability as the overall SD of the $R R$ interval divided by the $R R$ interval itself. $^{5}$

(g) The difference between the RR interval at the time the Doppler recording was made and the minimum value recorded during the 24 hours was noted.

\section{DATA ANALYSIS}

Values are expressed as mean (SD). The statistical significance of differences between mean values was examined by Student's $t$ test. Linear regression was performed by the method of least squares, and differences between groups compared by Fisher's exact probability test.

\section{Results}

HAEMODYNAMIC MEASUREMENTS

In the patients with dilated cardiomyopathy, left ventricular cavity size was considerably increased (to $7(0.7) \mathrm{cm}$ ) and shortening fraction was reduced to $14 \%(6 \%)$. Mean heart rate, derived from the 24 hour tapes, was slightly faster in the patients, although the difference from the control group was not significant. Isovolumic relaxation time varied widely, from -40 to $150 \mathrm{~ms}$. The mean duration of mitral regurgitation was $375(60)$ ms and filling time varied widely between 90 and $740 \mathrm{~ms}$.

\section{HEART RATE VARIABILITY}

Normal subjects

The table shows data for heart rate variability values in full. The SD of the RR interval and the index SDANN were closely related to one another $(r=0.99)$. Both the overall SD of the RR interval $(r=0.63)$ and also the SDANN index $(r=0.54)$ correlated significantly with the RR interval itself, but the coefficient of variation of the $R R$ interval did not. Correlation between $R R$ interval and the three indicators of short-term variability was much lower (RMSSD, $r=0.44$; PNN $50, r=0.38 ; S D$ index, $r=0.33, p=N S$ for all).

\section{PATIENTS}

The table shows that the mean $R R$ interval over the 24 hours in the patients did not differ significantly from that in the controls. Also, mean RR interval in individual patients correlated closely with that at the time the echocardiographic measurements were made. There was, however, no significant correlation between mean RR interval and the measures of short term variability-namely, the SD, RMSSD, and PNN 50 indices.

There were major differences in overall variability between patients. The simplest measure, the SD of the RR interval showed a wide range of values, although the mean was significantly less than normal. Values in individual patients correlated closely with $R R$ interval $(r=0.82)$. Unlike the normal finding, however, this correlation was also strong 

between left ventricular filling time and the $S D$ of the $R R$ interval for patients with dilated cardiomyopathy. A short filling period is associated with reduced heart rate variability.
Figure 1 Relation

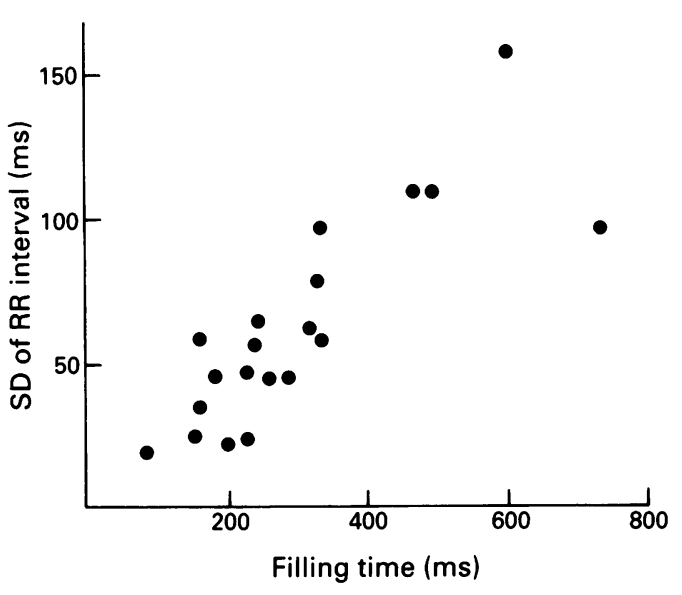

$(r=0.69)$ for the coefficient of variation. The other indicator of long-term variability, the SDANN, behaved similarly. It correlated closely with overall SD $(r=0.98)$, and again showed pronounced dependence on the RR interval $(r=0 \cdot 81)$.

The measures of short-term heart rate variability were much less abnormal in patients with dilated cardiomyopathy. Mean values of the SD, the RMSSD, and the PNN50 indices did not differ significantly from normal, although overall variability between patients was a little greater, particularly for SD. The RMSSD and the PNN50 correlated so closely with one another $(r=0.99)$, as to suggest that they reflected virtually identical aspects of heart rate variability in the patients we studied.

\section{CORRELATION WITH CLINICAL VARIABLES}

In the patients with dilated cardiomyopathy, heart rate variability did not correlate with age, left ventricular end diastolic dimension, or shortening fraction, or with the duration of the functional mitral regurgitation. There was some association with isovolumic relaxation time $(r=0.64$ for the SD of the RR interval and $r=0.62$ for SDANN). The major associations, however, were with left ventricular filling time $(r=0.81$ for SD (fig 1), and $r=$ 0.79 for SDANN). Filling time also correlated with the coefficient of variation of the RR interval $(r=0.70)$.

To investigate the mechanism of loss of variability in more detail, we took the difference between the $R R$ interval at the time the

Figure 2 Relation, in patients, between left ventricular filling time and the shortest $R R$ interval recorded during the 24 hours relative to that recorded during the echocardiogram (maximum difference in RR intervals). The shorter the filling time, the less is the difference between resting $R R$ interval and the shortest value recorded during the 24 hours. echocardiographic measurements were made and the minimum value throughout the 24 hours, giving a measure of the ability of the patient to increase heart rate on exercise. Again, this fall in $R R$ interval correlated closely with resting left ventricular filling time $(r=-0.92$, fig 2$)$. The slope of the regression line did not differ significantly from unity, nor the intercept from zero. This implies that the capacity of heart rate to increase with exercise or other metabolic demand can be directly predicted from resting left ventricular filling time. This capacity is greatly reduced when resting filling time is short.

\section{Discussion}

Dilated cardiomyopathy is one cause of the clinical syndrome of heart failure. It has a poor prognosis, so that on the basis of previous studies, ${ }^{10}$ reduced heart rate variability would be expected. Our patients were defined echocardiographically: all therefore had left ventricular cavity dilatation and reduced shortening fraction. Filling pressure was not measured directly, but in any individual case, this will have varied with diuretic and other treatment. Our relatively homogeneous group of patients will therefore have differed from those in whom the diagnosis of heart failure was based on the haemodynamic criteria such as a low cardiac index or a high filling pressure.

In our patients with dilated cardiomyopathy, the extent of the variability of heart rate differed greatly between patients. In some it was effectively normal, whereas in others it was greatly reduced. This reduction followed a characteristic pattern in that it was almost completely expressed in the simple measure of the SD of the RR interval between normally conducted beats. Similar information came from the SDANN index, the SD of the five minute means throughout the 24 hours, reflected long-term variability. The indices of short-term variability were not significantly different from normal.

By echocardiographic criteria, all the patients had severe left ventricular disease, so the wide scatter of heart rate variability between patients was unexpected. These large differences between patients could not be explained on the basis of age, cavity size, or shortening fraction. There was weak correlation with isovolumic relaxation time, which in these patients not only reflects left ventricular end diastolic pressure ${ }^{11}$ but also effectively determines left ventricular filling pattern. The shorter the isovolumic relaxation time, the greater is the preponderance of early diastolic filling and the smaller is that component occurring with left atrial systole. The most striking correlation of variability in heart rate, however, was with heart rate. This was much greater than has previously been reported in patients with heart failure selected on the basis of clinical state, cardiac output, or filling pressure, ${ }^{45}$ and still persisted in our study when the SD of the RR interval was normalised by dividing by the RR interval itself - 
that is, when the coefficient of variation was used.

This characteristic pattern of variability in heart rate needs to be explained. It may have simply reflected the illness of our patients, but this does not explain why we found the effect of heart rate to be so much stronger than in previous studies. Measuring filling pressure or diastolic filling pattern directly might have allowed us to predict the extent of variability in individual patients. We have previously shown, however, that isovolumic relaxation time correlates with both of these variables, yet in the present study its association with heart rate variability was much less than that of the RR interval. In patients with dilated cardiomyopathy, the period of forward flow across the mitral valve, as assessed by pulsed Doppler, is determined not by the time that the mitral valve cusps are open, but by the period between the pulses of mitral regurgitation. ${ }^{9}$ As the duration of the mitral regurgitation alters little with changing heart rate, a fall in the $R R$ interval leads to a disproportionate shortening of the time available for left ventricular filling. When this falls below $200 \mathrm{~ms}$, the normal $\mathrm{E}$ and $\mathrm{A}$ waves are replaced by a single summation pulse of forward flow. ${ }^{12}$ Any further fall in filling time thus reduces stroke volume or causes left atrial pressure to rise, either because of the increased pressure gradient necessary to accelerate and decelerate the same volume of blood across the valve in a shorter time, or to maintain filling into a ventricle that is still relaxing at the end of one systole or has already begun to contract at the onset of the next. Any further increase in heart rate thus carries a physiological penalty: either a fall in stroke volume or an increase in left atrial pressure. We suggest that this mechanical restraint on short $R R$ intervals directly limits the extent of heart rate variability. There was close correlation between resting filling time and the extent to which the RR interval fell at the time of maximum heart rate. These interrelations between filling time, minimum $R R$ interval, and the extent of heart rate variability have not previously been recorded in patients with heart failure. We suggest that they reflect the constraints on left ventricular filling time caused by the presence of functional mitral regurgitation.

Our study has some potential limitations. Echocardiographic Doppler studies reflected only a single short, and not necessarily representative period during the 24 hours. It is obviously possible that major changes in haemodynamics occur at other times of day, possibly with exercise or sleep, which are not reflected in a single resting study. We did note, however, a close correlation between the average $R R$ interval throughout the 24 hours and the values recorded during the study itself, with no consistent difference between the two. As the other variables we measured were related to heart rate, we are reassured that our echocardiographic measurements were representative. We were unable to dissociate effects of heart rate com- pletely from those of filling time. This was not unexpected, because the two are so closely associated in disease. Methods of altering filling time at constant heart rate are complex and not easily adaptable to a study such as this. Nevertheless, the association of heart rate variability with filling time, if not definitely proved, seems suggestive. Finally, the mechanism we have proposed for reduced variability in heart rate depends not only on a minimum value of the $R R$ interval imposed by left ventricular filling time, but also on a tendency for sinus tachycardia. Presumably this tendency is on the basis of a fixed stroke volume or increased reflex activity the origin of which we did not define, but which is likely to reflect some overall aspect of the severity of the condition.

In conclusion, patients with dilated cardiomyopathy show a characteristic pattern of reduced variability in heart rate. We suggest that the close relation to the $R R$ interval reflects the importance of a short left ventricular filling time. This raises the possibility of a mechanical rather than a purely reflex basis. We appreciate, however, that in these patients individual mechanisms can rarely be isolated. Sinus tachycardia may be an index of increased sympathetic activity or of a small fixed stroke volume. The presence of left ventricular disease itself is likely both to reduce variability in heart rate and impair prognosis. We have previously suggested that a short filling time is basically unstable, and may, of itself, provide a mechanism for sudden death on a purely haemodynamic basis. ${ }^{12}$ It would be perhaps more realistic to accept the complexity of these interrelations, and adopt a more general approach to intervention. Rather than trying to isolate individual disturbances for treatment, one should accept that any simple intervention will cause a series of possibly contradictory changes, and thus aim at defining the new balance that has resulted. Estimates of heart rate variability seem to provide an additional independent means of assessing such patients, and to complement standard haemodynamic and imaging approaches.

1 Martin GJ, Magid NM, Myers G, Barnett PS, Schaad JW, Weiss JS, et al. Heart rate variability and sudden death secondary to coronary artery disease during ambulatory electrocardiographic monitoring. Am $\mathcal{f}$ Cardiol 1987;60: 86-9.

2 Kleiger RE, Miller JP, Bigger Jr JT, Moss AJ. Decreased heart rate variability and its association with increased mortality after acute myocardial infarction. $A m \mathcal{f}$ Cardiol 1987;59:256-62.

3 Saul JP, Arai Y, Berger RD, Lilly LS, Colucci WS, Cohen RJ. Assessment of autonomic regulation in chronic conR. Assessment of autonomic regulation in chrysic $A m \mathfrak{f}$ Gestive heart failure by heart rate spectral analysis. Am

4 Marin-Neto JA, Pintya AO, Gallo Jr L, Maciel BC. Abnormal baroreflex control of heart rate in decompensated congestive heart failure and reversal after compensation. Am f Cardiol 1991;67:604-10.

5 Van Hoogenhuyze D, Weinstein N, Martin GJ, Weiss JS, Schaad JW, Sahyouni XN, et al. Reproducibility and relation to mean heart rate of heart rate variability in normal subjects and in patients with congestive hear failure secondary to coronary artery disease. $A m \mathcal{F}$ Cardiol 1991;68:1668-76.

6 Goldstein RE, Beiser GD, Stampfer M, Epstein SE. Impairment of autonomically mediated heart rate control in patients with cardiac dysfunction. Circ Res 1975; 36:571-8. 
7 Pomeranz B, Macaulay RJB, Caudill MA, Kutz I, Adam $\mathrm{D}$, Gordon D, et al. Assessment of autonomic function in humans by heart rate spectral analysis. Am $\mathcal{f}$ Physio 1985;248: H151-3.

8 Cripps TR, Malik M, Farrell TG, Camm AJ. Prognostic value of reduced heart rate variability after myocardial infarction: clinical evaluation of a new analysis method. Br Heart f 1991 ;65:14-9.

$9 \mathrm{Ng} \mathrm{KSK}$, Gibson DG. Impairment of diastolic function by shortened filling period in severe left ventricular disease. Br Heart $\mathcal{F}$ 1989;62:246-52.
10 Franciosa JA, Wilen M, Ziesche S, Cohn JN. Survival in men with severe chronic left ventricular failure due either to coronary heart disease or idiopathic dilated cardiomyopathy. Am $\mathcal{f}$ Cardiol 1983;51:831-6.

11 Mattheos M, Shapiro E, Oldershaw PJ, Sacchetti R Gibson DG. Non-invasive assessment of changes in left ventricular relaxation by combined phono-, echo-, and mechanography. Br Heart f 1982;47:253-60.

$12 \mathrm{Ng} \mathrm{KSK}$, Gibson DG. Relation of filling pattern to diastolic function in severe left ventricular disease. $\mathrm{Br}$ Heart f 1990;45:209-14. 